\title{
Der neue Datenschutz
}
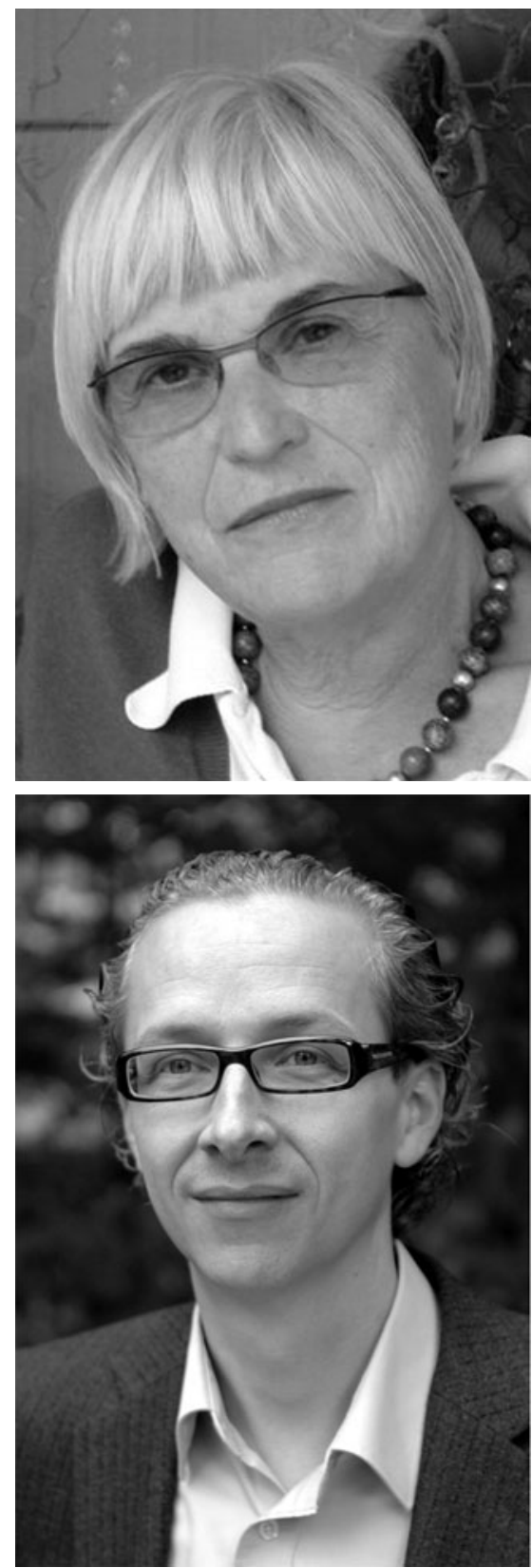

Die digitalen Prozesse steuern unser Leben und Denken in atemberaubender Weise und rund um den Globus. NSA, Google, Facebook und Co haben sich in diese Entwicklung eingeklinkt; sie erfassen, überwachen und steuern unendlich viele persönliche Datenströme, häufig durch den Einsatz von Algorithmen. Viele Menschen wissen nicht, was mit ihren persönlichen Daten geschieht, die sie per Twitter, Social Media und Smartphone weiterreichen. Der kürzlich verstorbene Herausgeber der FAZ, Frank Schirrmacher, ist dieser Entwicklung nachgegangen und stellt in seinem Werk „Payback“ (2011, S. 15) sinngemäß die Frage nach dem „Wer frisst wen in der digitalen Gesellschaft?".

Im europäischen Datenschutzrecht steht eben diese Frage zur Entscheidung an. Mit der geplanten supranationalen EU-Datenschutz-Grundverordnung setzen sich Kommission, Rat und Parlament im derzeit laufenden Trilog dafür ein, dass der $\mathrm{Ci}$ toyen die Selbstbestimmung und Kontrolle über seine Daten zurückgewinnt. Heiko Maas befasst sich mit den Grundzügen, den bereichsspezifischen Regelungen und den derzeitigen Perspektiven der Verordnung, an deren Gestaltung er von deutscher Seite als Bundesjustizminister maßgeblich beteiligt ist. Der "deutsche" Richter am EuGH, Thomas von Danwitz, behandelt die jüngste Rechtsprechung des Gerichtshofs zu den Gefährdungen des Privatlebens; er geht auf die Konsequenzen ein, die sich daraus für das Recht und die Rechtsprechung in den Mitgliedstaaten ergeben, und spricht vor dem Hintergrund der Google Spain-Entscheidung vor allem auch den Konflikt zwischen Datenschutz und Kommunikationsfreiheit an. Sabine LeutheusserSchnarrenberger setzt diese Überlegungen fort und behandelt das bemerkenswerte EuGH-Urteil zu Google Spain vom 13. Mai 2014, dessen herausragende Bedeutung sie nicht auf das viel zitierte Recht auf Vergessenwerden reduziert, sondern vor allem auch darin sieht, dass ", mit den Mitteln des Rechts" Würde, Freiheit und Selbstbestimmung des Einzelnen vor den unbegrenzten Möglichkeiten digitaler Kommunikation geschützt werden. Und auch im Beitrag von Johannes Caspar steht die Google SpainEntscheidung im Mittelpunkt; als Hamburgs Datenschutzbeauftragter zuständig für Facebook in Deutschland erläutert er die Konsequenzen, die sich aus der EuGH-Entscheidung für die Kontrolle dieses sozialen Netzwerks ergeben.

Hauke Gärtner und Dennis-Kenji Kipker setzen sich mit dem aktuellen Gesetzesentwurf für eine Vorratsdatenspeicherung auseinander und entwickeln Lösungsansätze, wie zentralen Kritikpunkten an diesem Entwurf begegnet werden kann. Maja Beisenherz beleuchtet in ihrem Beitrag die Schnittstelle zwischen Kartell- und Datenschutzrecht und plädiert dafür, künftig in die Fusions- und Missbrauchskontrolle auch datenschutzrechtliche Maßstäbe einfließen zu lassen. Unter dem Aspekt des Medienprivilegs ist die Google Spain-Entscheidung schließlich auch Thema der Ausführungen von Volker Schumacher und Jochen Spindler; die beiden Autoren gehen am Beispiel der Suchmaschinen der Frage nach, ob und wie über das Medienprivileg ein gerechter Ausgleich zwischen Kommunikationsfreiheit und Datenschutz hergestellt werden kann. Auf welch verschlungene Art und Weise sich staatliche Datenbanken ihren Weg ans Tageslicht bahnen können, zeichnet Burkhard Schafer in seinem Beitrag am Beispiel der derzeitigen Pläne für ein schottisches National IDSystem nach. Ulrich Möncke schließlich zeigt die technischen und rechtlichen Rahmenbedingungen auf, die für den Einsatz von cloudbasierten Werkzeugen in der Hochschullehre gelten.

\section{Marie-Theres Tinnefeld \\ Benedikt Buchner}

